\title{
FACTORES PREDICTORES DEL DESEMPEÑO DE UN GRUPO DE DOCENTES UNIVERSITARIOS
}

\section{PREDICTIVE FACTORS OF TEACHING PERFORMANCE IN A GROUP OF UNIVERSITY PROFESSORS}

\author{
Prenam Abeh \\ Universidad de Montemorelos, México \\ dinaabeh@gmail.com \\ https://orcid.org/0000-0003-4307-044X \\ Manuel Muñoz Palomeque \\ Universidad de Montemorelos, México \\ manuelmp@um.edu.mx \\ https://orcid.org/0000-0003-0816-8169
}

\begin{abstract}
RESUMEN
Este estudio tuvo como objetivo determinar si la motivación laboral, el clima organizacional, la capacitación docente y la percepción del grado de cumplimiento de la misión de la institución son predictores significativos del desempeño docente autopercibido por 110 docentes que representan el $58 \%$ del plantel de profesores de una universidad del noreste de México. Mediante el análisis de regresión lineal múltiple, se encontró que las cuatro variables incluidas en el análisis explican un $80.7 \%$ de la varianza del desempeño docente. Se pudo inferir estadísticamente que existió una influencia lineal positiva y significativa. Se encontró que las variables motivación laboral, capacitación docente y misión institucional fueron predictores significativos del desempeño docente, pero el aporte del clima organizacional no resultó significativo. Además, el mejor predictor fue la capacitación docente $(\beta=.623)$. Se concluye que, en la medida en que los docentes mejoren su nivel de motivación laboral, reciban mayor capacitación docente e interioricen mejor la misión institucional, se incrementará de manera importante su desempeño laboral.
\end{abstract}

Palabras clave: motivación laboral, clima organizacional, capacitación docente, misión institucional, desempeño docente

\section{ABSTRACT}

The purpose of this study was to determine whether work motivation, organizational climate, teacher training, and the perception of the degree of fulfillment of the institution's mission are significant predictors of the self-perceived teaching performance of 110 teachers who represent $58 \%$ of the faculty members from a university in Northeastern Mexico. Through multiple linear regression analysis, it was found that the four variables included in the analysis explain $80.7 \%$ of the variance of 
teacher performance. Therefore, It could be statistically inferred there was a positive and significant linear influence. It was found that the variables work motivation, teacher training, and perception of the institutional mission were significant predictors of teacher performance, but the contribution of the organizational climate was not significant in the model. Furthermore, the best predictor was teacher training $(\beta=.623)$. It is concluded that, to the extent that teachers improve their work motivation, receive more teacher training, and better internalize the institutional mission, their work performance will increase significantly.

Keywords: work motivation, organizational climate, teaching training, institutional mission, teaching performance

\section{Introducción}

México, como otros países del mundo, enfrenta una diversidad de problemas para lograr un resultado óptimo en el ámbito educativo. En algunos países, el nivel de rendimiento académico de los estudiantes es muy bajo; en otros, los docentes rinden muy poco, al igual que sus alumnos, posiblemente debido a la poca evaluación de su desempeño. $\mathrm{Al}$ respecto, Pedraza et al. (2010) señalan que el desempeño de los profesores se ha considerado como uno de los aspectos más importantes y determinantes en el campo educativo. Por tal razón, actualmente las autoridades educativas están ocupadas en medir y mejorar el desempeño de los docentes. Una manera de medir el desempeño docente es considerar la opinión de los alumnos. En un estudio realizado en una institución universitaria, se evaluó el desempeño de los docentes por parte de los alumnos y esta obtuvo una calificación alta (Rodríguez Cavanerio y Coelho de Torres, 2018). Otra manera es considerar la percepción del mismo docente. Desde esta perspectiva, el objetivo que guio el presente estudio fue observar la motivación laboral, el clima organizacional, la capacitación docente y la percepción del grado de cumplimiento de la misión de la institución como predictores del desempeño laboral percibido por los docentes de una universidad en el noreste de México.

\section{Precisiones conceptuales}

Motivación laboral. La motivación laboral es un proceso interno y propio de cada persona que refleja la interacción que se establece entre el individuo y el mundo, en este caso, su trabajo (González Serra, 2008). La motivación en el trabajo es la fuerza, tanto interna como externa, que hace que la persona dé lo mejor de sí (Sum Mazariegos, 2015). Es una fuerza que da energía y dirige y mantiene el comportamiento de las personas (Madrigal Torres, 2009).

Clima organizacional. El clima organizacional es un constructo multidimensional que considera el tamaño de la organización, los modos de comunicación y el estilo de liderazgo, entre otros aspectos (Rodríguez Montufar, 2020). Mujica de González y Pérez de Maldonado (2007) consideran al clima organizacional como "el conjunto de percepciones compartidas que las personas se forman acerca de la realidad del trabajo en la organización, donde concurren los componentes sociales (políticas, normas, reglamentos, desempeño de roles, 
funcionamiento de grupos)” (p. 294). En síntesis, el clima organizacional se refiere tanto a una percepción individual como al ambiente interno que se vive en una institución.

Capacitación docente. Edelfelt y Lawrence (1975) definen la capacitación docente como "el continuo desarrollo del personal, como cualquier tipo de actividad de desarrollo profesional que hace un docente después que reciba su certificado de docencia y después del comienzo de la práctica profesional" ( $p$. 1). Además, Lowenthal (1981) considera que la educación profesional continua es la educación y el entrenamiento "más allá de la básica profesión de la licenciatura, y también como un entrenamiento que se toma después de haber salido de alguna carrera para capacitar a los profesionales para que puedan poner sus conocimientos en práctica" (p. 519). La capacitación docente, en este estudio, puede ser considerada como el aporte de cada actividad que se realiza con el fin de aumentar el nivel intelectual o la capacidad de desarrollar nuevas estrategias de enseñanza aprendizaje.

Misión institucional. La misión institucional está conformada por las metas institucionales y el resultado intermedio que se quiere alcanzar en un tiempo determinado (Chiavenato, 2013). Es la razón de ser de la existencia de una institución, porque define lo que pretende cumplir en el entorno o sistema social en el que actúa (Sallenave, 2010). En este estudio se midió en qué medida los docentes participantes percibían la naturaleza única y peculiar que identifica a la institución en la que laboran y cómo ella orienta sus fines y su accionar en la sociedad.

Desempeño docente. El desempeño docente se refiere al nivel con el que los profesores llevan sus estrategias didácticas en el salón de clases (Wu et al., 2019). El desempeño docente constituye un indicador muy relevante en la evaluación de la calidad educativa de las instituciones de enseñanza superior (Carlucci et al., 2019).

\section{Relación entre variables}

Motivación laboral y desempeño docente. Castro Aponte (2017) encontró una relación directa entre la motivación y el desempeño laboral. También, Burga Vásquez y Wiesse Eslava (2018) encontraron una relación significativa de grado moderado $(r=0.604)$ entre la motivación y el desempeño del personal administrativo en la empresa agroindustrial de la región Lambayeque, Perú. De acuerdo con Ayala Gutiérrez y Pajuelo Fernández (2018), la motivación tiene una relación directamente proporcional con el desempeño laboral de los trabajadores del juzgado de subespecialidad comercial.

Específicamente en el ámbito educativo, Moncada Herrera (2019) encontró que la motivación laboral en docentes universitarios tiene una relación directa con el reconocimiento económico que esperan recibir, mientras otros estudios observaron una relación significativa entre la motivación y el desempeño laboral en los docentes (Callata Gallegos y Fuentes López, 2018; Canchari Fierro, 2017).

\section{Clima organizacional y desempe-} ño docente. Hay diversos estudios que han observado una relación significativa positiva entre el clima organizacional y el desempeño laboral (Llorrén Cáceres, 2018; Melo, 2020; Quintero et al., 2008). Igualmente, en el ámbito específicamente educativo, se encontró una relación significativa positiva entre el 
clima organizacional y el desempeño docente (Paul, 2016).

La capacitación y el desempeño docente. Hay evidencia de la relación que existe entre la capacitación del docente y su desempeño. Por ejemplo, Cutimbo Estrada (2008) mostró una correlación directa entre el nivel de capacitación docente y el rendimiento académico de los estudiantes, influenciado por el desempeño docente. La investigación de Ramírez Amado (2014), sobre la capacitación docente y desempeño laboral de los empleados de la Universidad Linda Vista, Chiapas, encontró que la capacitación docente tiene una influencia lineal positiva y significativa sobre el desempeño laboral. También Al-Mzary et al. (2015), hallaron una relación positiva entre la capacitación eficaz y el desempeño laboral de los empleados. Contrario a lo anterior, García Oquendo et al. (2014), en estudio con profesores universitarios, no encontraron relación entre los cursos realizados, la capacitación recibida y el desempeño docente.

\section{Misión institucional y desempeño} docente. La misión institucional, por definición, orienta las actividades que se desarrollan en toda la organización. Sin embargo, no siempre tiene un impacto significativo sobre el desempeño del personal. En el estudio de Del Valle López (2016) y Charnichart Castañarez (2018), la percepción del grado de cumplimiento de la misión institucional tuvo un impacto significativo sobre la percepción del grado del desempeño laboral. Sin embargo, en el estudio de Dharmadasa et al. (2012), no se observó impacto alguno de la misión sobre el desempeño de los empleados.

Modelo teórico del estudio. Desde la perspectiva teórica, la Figura 1 ilustra el modelo considerado en el presen- te estudio, donde la motivación laboral, el clima organizacional, la capacitación docente y la misión institucional son predictores significativos del desempeño docente en una universidad del noreste de México.

\section{Figura 1}

Modelo teórico propuesto

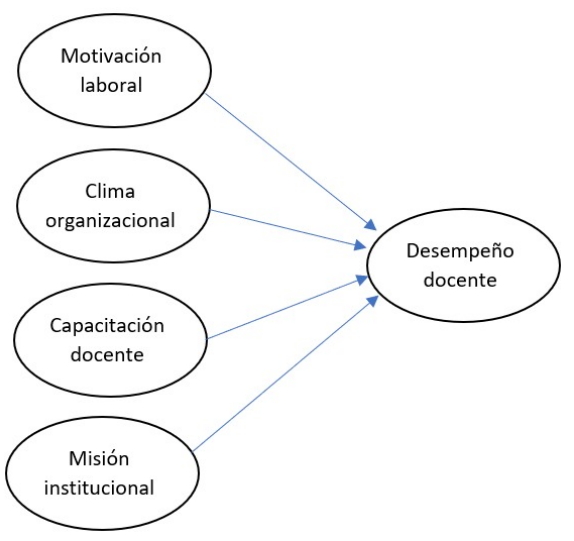

\section{Método}

Este estudio tuvo un enfoque cuantitativo, transversal, descriptivo y predictivo.

\section{Participantes}

Se realizó un muestreo no probabilístico, por conveniencia. La muestra estuvo conformada por 110 docentes, que representa el $57.9 \%$ de la población $(N$ $=190) ; 59.1 \%$ de ellos fueron del sexo femenino. La edad promedio fue de 48.1 años. La mayoría manifestó tener $11 \mathrm{o}$ más años de servicio (71.8\%). En cuanto al nivel académico, el $47.3 \%$ dijo tener el grado de maestría.

\section{Instrumentos}

Motivación laboral. Se utilizó una escala para medir la motivación laboral creada por Enríquez Loredo (2014). Consta de 19 ítems, valorados con una escala tipo Likert de cinco puntos, cuyo 
rango se extiende desde pésimo hasta excelente. El instrumento obtuvo un alfa de Cronbach de .848 .

Clima organizacional. Para medir el clima organizacional, se utilizó un cuestionario elaborado por Juárez-Adauta (2012), formado por 20 ítems, valorados con una escala tipo Likert de cinco puntos, cuyo rango se extiende desde pésimo hasta excelente. La consistencia interna del instrumento, medida con el alfa de Cronbach, obtuvo un valor de .943 .

Capacitación docente. La capacitación docente fue medida con un instrumento diseñado por Ramírez Amado (2014), que cuenta con 15 ítems valorados con una escala Likert de cinco opciones, que va desde totalmente en desacuerdo hasta totalmente de acuerdo. La confiabilidad, medida con el coeficiente alfa de Cronbach, fue de .846.

Misión institucional. La variable misión institucional fue medida utilizando el cuestionario elaborado por Del Valle López (2016), formado por 23 ítems que fueron valorados con una escala $\mathrm{Li}$ kert de cinco puntos, que va desde pésimo a excelente. El instrumento obtuvo un alfa de Cronbach de .972.

Desempeño docente. Se utilizó un instrumento creado por Enríquez Loredo (2014) para medir el desempeño docente. Consta de 48 ítems valorados con una escala Likert de cinco puntos, que se extiende desde totalmente en desacuerdo hasta totalmente de acuerdo, con un coeficiente alfa de Cronbach igual a 943 .

Los datos se obtuvieron de manera virtual con la aplicación MailChimp.

\section{Resultados}

De acuerdo con la Tabla 1, se observa que el constructo mejor evaluado fue el desempeño docente autopercibido, mientras el que obtuvo una menor valoración fue el clima organizacional.

\section{Tabla 1}

Estadísticos descriptivos de los constructos del estudio

\begin{tabular}{lcc}
\hline \multicolumn{1}{c}{ Constructos } & $M$ & DE \\
\hline Motivación laboral & 4.687 & .2941 \\
Clima organizacional & 4.015 & .6794 \\
Capacitación docente & 4.687 & .2966 \\
Misión institucional & 4.167 & .7305 \\
Desempeño laboral & 4.809 & .2255 \\
\hline
\end{tabular}

\section{Supuestos de regresión}

En esta investigación se consideraron los siguientes cinco supuestos de la regresión lineal múltiple: linealidad del fenómeno, normalidad de residuos estandarizados, independencia de los términos de error, varianza constante del término de error, es decir, homocedasticidad e inexistencia de multicolinealidad (Hair et al., 1999).

El primer criterio analizado fue la linealidad de las cuatro variables independientes con la variable dependiente. Se observó en los gráficos de dispersión que existe una relación lineal positiva donde los puntos tienden a formar una línea recta ascendente (ver Figura 2).

\section{Figura 2}

Linealidad de las variables independientes con la variable dependiente

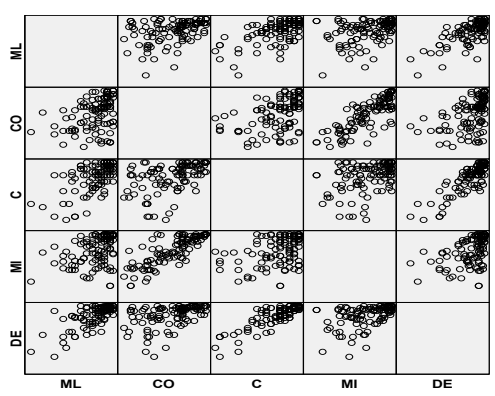


El segundo criterio que se probó fue la normalidad de los residuos (ver Figuras 3 y 4). Se utilizó el estadístico de Kolmogorov-Smirnov y se observó que la distribución de los residuos es normal $(Z=.052, p=.200)$.

\section{Figura 3}

Histograma de normalidad Histograma

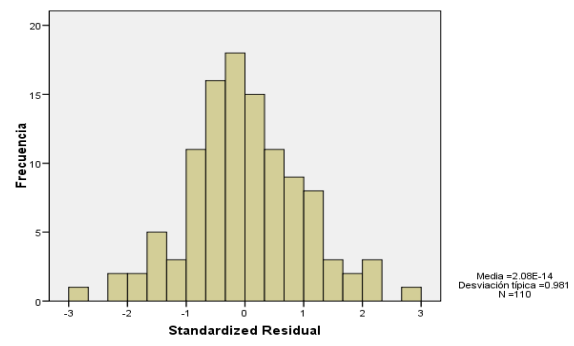

\section{Figura 4}

Representación gráfica de normalidad de los datos

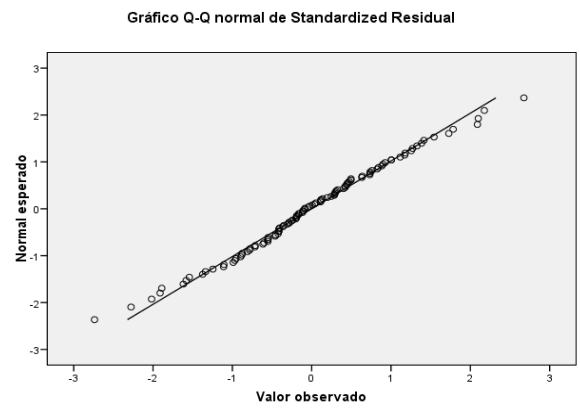

En el tercer criterio se probó la independencia de los errores. Se utilizó la prueba Durbin-Watson, que es una prueba estadística que contrasta la presencia de autocorrelación en los residuos de una regresión. El valor obtenido muestra que no existe autocorrelación de los residuos $\left(R=.901, R^{2}=.813, R^{2}\right.$ corregida $=.807$, estadístico Durbin-Watson $=2.011$ ).

El cuarto criterio fue la homocedasticidad, utilizando el gráfico del valor predicho estandarizado y el valor del residuo estandarizado. Se observó que no hay relación lineal entre los residuos, por lo tanto, los errores tienen varianzas iguales (ver Figura 5).

\section{Figura 5}

Representación gráfica de la homocedasticidad

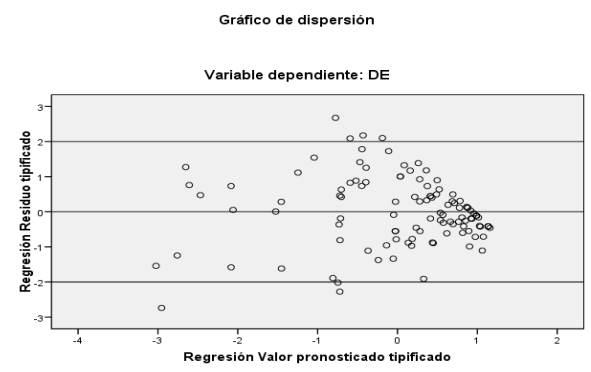

El último criterio fue comprobar la inexistencia de multicolinealidad; es decir, se buscó que las variables independientes no estén correlacionadas entre sí. De acuerdo con los factores de inflación de la varianza (VIF) observados (FIV ${ }_{\mathrm{ML}}$ $=1.677, \mathrm{FIV}_{\mathrm{CD}}=1.713, \mathrm{FIV}_{\mathrm{MI}}=1.249$ ), se concluye que, por ser los valores menores a 4 , los constructos no presentan colinealidad.

\section{Prueba del modelo}

Se procuró observar si la motivación laboral, el clima organizacional, la capacitación docente y la misión de la institución son predictores significativos del nivel de desempeño docente autopercibido por los docentes participantes.

Al realizar el análisis de regresión lineal múltiple, se encontró que el mejor modelo predictor estuvo formado por la motivación laboral, la capacitación docente y la misión institucional, que explican el $80.7 \%$ de la varianza del desempeño docente $\left(R=.901, R^{2}=.812, R^{2}\right.$ corregida $=.807)$. 
Los valores de los coeficientes no estandarizados $(B k)$ permiten construir la siguiente ecuación de regresión (ver Tabla 2) utilizando el método de mínimos cuadrados: desempeño docente $=1.341$ +.234 (motivación laboral) + .474 (capacitación docente) +.034 (misión ins- titucional).

El mejor predictor del desempeño docente fue la capacitación docente $(\beta$ $=.623$ ). Por otro lado, se excluyó del modelo la variable clima organizacional ( $p=.223)$, porque su aporte no resultó significativo.

\section{Tabla 2}

\begin{tabular}{|c|c|c|c|c|c|c|c|c|}
\hline \multirow[t]{2}{*}{ Modelo } & & \multicolumn{2}{|c|}{$\begin{array}{c}\text { Coeficientes } \\
\text { no } \\
\text { estandarizados }\end{array}$} & \multirow[t]{2}{*}{$\begin{array}{c}\text { Coeficientes } \\
\text { estandarizados }\end{array}$} & \multirow[t]{2}{*}{$t$} & \multirow[t]{2}{*}{$\mathrm{p}$} & \multicolumn{2}{|c|}{$\begin{array}{c}\text { Estadísticos de } \\
\text { colinealidad }\end{array}$} \\
\hline & & $b$ & $\begin{array}{l}\text { Error } \\
\text { típ. }\end{array}$ & & & & Tolerancia & FIV \\
\hline \multirow[t]{4}{*}{1} & (Constante) & 1.341 & .170 & & 7.894 & .000 & & \\
\hline & $\begin{array}{l}\text { Motivación } \\
\text { laboral }\end{array}$ & .234 & .042 & .305 & 5.585 & .000 & .596 & 1.677 \\
\hline & $\begin{array}{l}\text { Capacitación } \\
\text { docente }\end{array}$ & .474 & .042 & .623 & 11.307 & .000 & .584 & 1.713 \\
\hline & $\begin{array}{l}\text { Misión } \\
\text { institucional }\end{array}$ & .034 & .015 & .112 & 2.371 & .020 & .801 & 1.249 \\
\hline
\end{tabular}

En el presente estudio se calculó el tamaño del efecto, de la regresión con el software GPower. Para calcular el tamaño del efecto se utilizó la sensibilidad como tipo de análisis y como parámetros un $\alpha$ de .05 , una potencia estadística de 0.99 , una muestra de 110 individuos $\mathrm{y}$ tres predictores. Al calcular el tamaño del efecto, se encontró un valor de 0.222 , considerado como una medida del tamaño del efecto medio alto $\left(f^{2}>\right.$ $\left..15 \mathrm{y} f^{2}<.35\right)$.

\section{Discusión}

En este estudio se encontró que la motivación laboral es predictor significativo del desempeño docente. Para lograr una educación de calidad se requiere que los docentes estén motivados, aunque no es el único factor que interviene. Nuestros resultados concuerdan con los de otros estudios (Ayala Gutiérrez y Pajuelo Fernández, 2018; Burga
Vásquez y Wiesse Eslava, 2018; Canchari Fierro, 2017; Castro Aponte, 2017; Moncada Herrera, 2019), que hallaron una relación directa entre la motivación y el desempeño laboral. Por lo tanto, de acuerdo con los resultados de nuestro estudio, se recomienda que las instituciones educativas utilicen estrategias que promuevan la motivación laboral de sus docentes.

El clima organizacional cumple un papel importante en el desempeño laboral en el ámbito educativo. Hay investigaciones que muestran que el clima organizacional es un predictor significativo del desempeño docente (Paul, 2016). Sin embargo, el nivel de clima organizacional percibido por los docentes, en conjunto con las otras tres variables independientes, no resultó ser un predictor significativo de su desempeño en el modelo, pero debido a su correlación altamente significativa con las demás 
variables predictoras, tiene un efecto predictor indirecto. Se observó una alta valoración en las relaciones interpersonales con jefes y compañeros de trabajo, $\mathrm{y}$ en el reconocimiento, el ambiente físico, el nivel de seguridad, las prestaciones de salud, la calidad moral y el apoyo para el desarrollo personal. El resultado obtenido también puede atribuirse a la importancia que los docentes le atribuyen a la filosofía, los valores y la misión institucional, que trascienden más allá del clima organizacional percibido.

Respecto de la capacitación docente, Edelfelt y Lawrence (1975) mencionan que la capacitación docente es, en pocas palabras, la actualización continua. Por lo tanto, cuando el empleado es capacitado, mejora el desempeño de sus funciones. En este estudio, la capacitación docente resultó ser un predictor significativo del desempeño docente, lo que concuerda con otros estudios llevados a cabo (Al-Mzary et al., 2015; Cutimbo Estrada, 2008; Ramírez Amado, 2014).

La misión institucional es la razón de ser de una institución. En el presente estudio la percepción del grado de su cumplimiento resultó ser un predictor significativo del desempeño docente, lo que concuerda con los resultados encontrados por Charnichart Castañarez (2018) y Del Valle López (2016), quienes encontraron una relación significativa entre la misión institucional y el desempeño de las organizaciones.

Partiendo del hecho de que la educación es un pilar para el crecimiento del ser humano y de la sociedad en general, debe considerarse todo estudio que pretenda proveer información que ayu- de a la mejora del proceso de enseñanza-aprendizaje.

Desde esta perspectiva, y con el propósito de comprender y apoyar el desempeño de los docentes desde una perspectiva empírica científica, el propósito de este estudio fue examinar el modelo teórico donde la motivación laboral, el clima organizacional, la capacitación docente y la misión de la institución son predictores del desempeño de los docentes.

Aunque hay múltiples estudios que proponen la influencia de estas variables sobre el desempeño de los docentes, no se encontraron estudios previos que analicen el modelo completo de esta investigación.

Los resultados de los análisis estadísticos proveen información para inferir que la motivación laboral, la capacitación docente y la misión institucional son predictores significativos del desempeño docente, de acuerdo con los datos provistos para esta muestra poblacional.

En síntesis, en la medida en que tanto los docentes como la institución utilicen estrategias que mejoren el nivel de motivación laboral, reciban más capacitación docente e interioricen la misión institucional, influirá de manera importante en su desempeño laboral. Sin embargo, la valoración de algunos elementos del clima organizacional que han sido percibidos en forma poco satisfactoria merece mayor atención. Con esto en contexto, se puede afirmar que la institución educativa tiene una gran responsabilidad de colaborar activamente con sus docentes $\mathrm{y}$, de esta forma, incrementar su nivel de desempeño y mejorar su calidad educativa.

\section{Referencias}

Al-Mzary, M. M. M., Al-rifai, A. D. y Al-Momany, M. O. E. (2015). Training and its impact on the performance of employees at Jordanian Universities from the perspective of employees: The case of 


\section{FACTORES PREDICTORES DEL DESEMPEÑO DOCENTE}

Yarmouk University. Journal of Education and Practice, 6(32), 128-140. http://www.rexe.cl/ojournal/ index.php/rexe/article/view/47/49

Ayala Gutierrez, T.M. y Pajuelo Fernández, S. O.(2018). La motivacióny su influenciaenel desempeño laboral de los colaboradores de los juzgados de subespecialidad comercial de la Corte Superior de Justicia de Lima, 2018 [Tesis de licenciatura, Universidad Tecnológica del Perú]. Respositorio Institucional, Universidad Tecnológica del Perú. https://repositorio.utp.edu.pe/handle/20.500.12867/2975

Burga Vásquez, G. y Wiesse Eslava, S. J. (2018). Motivación y desempeño laboral del personal administrativo en una empresa agroindustrial de la región Lambayeque [Tesis de maestría, Universidad Católica Santo Toribio de Mogrovejo]. DSpace. http://tesis.usat.edu.pe/xmlui/handle/20.500.12423/1108

Callata Gallegos, Z. E. y Fuentes López, J. D. (2018). Motivación laboral y desempeño docente en la Facultad de Educación de la UNA-Puno. Revista de Investigaciones de la Escuela de Posgrado, 7(2), 592-597. https://doi.org/10.26788/riepg.2018.2.83

Canchari Fierro, Y. E. (2017). Motivación laboral y desempeño laboral en docentes de la Facultad de Enfermería de la Universidad Nacional de Huancavelica [Tesis de maestría, Universidad Nacional de Huancavelica]. Repositorio Intitucional, Universidad Nacional de Huancavelica. https://repositorio unh.edu.pe/handle/UNH/1394

Carlucci, D., Renna, P., Izzo, C. y Schiuma, G. (2019). Assessing teaching performance in higher education: A framework for continuous improvement. Management Decision, 57(2), 461-479. https:// doi.org/10.1108/MD-04-2018-0488

Castro Aponte, P. C. (2017). Motivación y desempeño laboral en los trabajadores de la Dirección Regional de Educación del Callao en el año 2016 [Tesis de maestría, Universidad César Vallejo]. Repositorio Institucional Digital, Universidad Cesar Vallejo. https://repositorio.ucv.edu.pe/ handle/20.500.12692/8520?show=full

Charnichart Castañarez, C. E. (2018). Modelo de asociación entre factores de la misión institucional y el desempeño laboral validado con directivos de Instituciones Adventistas en Maryland, Estados Unidos [Tesis doctoral, Universidad de Montemorelos]. DSpace. http://dspace.biblioteca.um.edu.mx/xmlui/ handle/20.500.11972/204

Chiavenato, I. (2013). Introducción a la teoría general de la administración (8a ed.). McGraw-Hill.

Cutimbo Estrada, P. M. (2008). Influencia del nivel de capacitación docente en el rendimiento académico de los estudiantes del Instituto Superior Pedagógico Público de Puno: caso de la Especialidad de Educación Primaria IX Semestre-2008 [Tesis de maestría, Universidad Nacional Mayor de San Marcos]. Cybertesis: Repositorio de Tesis Digitales. https://cybertesis.unmsm.edu.pe/ handle/20.500.12672/2395

Del Valle López, J. A. (2016). Modelo asociativo entre factores determinantes del desempeño organizacional y la satisfacción de los públicos en instituciones educativas [Tesis doctoral, Universidad de Montemorelos]. DSpace. http://dspace.biblioteca.um.edu.mx/xmlui/handle/20.500.11972/195

Dharmadasa, P., Maduraapeurma, Y. y Herath, S. K. (2012). Mission statements and company financial performance revisited. International Journal of Managerial and Financial Accounting, 4(3), 314-324. https://doi.org/10.1504/IJMFA.2012.047855

Edelfelt, R. A. y Lawrence, G. (1975). In-service education: The state of the art in rethinking in-service education. NEA.

Enríquez Loredo, P. C. (2014). Motivación y desempeño laboral de los empleados del Instituto de la Visión en México [Tesis de maestría, Universidad de Montemorelos]. DSpace. http://dspace .biblioteca.um.edu.mx/xmlui/handle/20.500.11972/99

Fernández Sotolongo, J., Berra Socarrás, M., Suárez Romero, M. y Hernández González, E. M. (2014). Nuevo modelo de formación de especialistas en Oftalmología generado por la Misión Milagro. Educación Médica Superior, 28(1), 26-34. http://ems.sld.cu/index.php/ems/article/view/332

García Oquendo, M. V., Rueda Fierro, I. A., Altamirano Cumbajín, J. P. y Gea Izquierdo, E. (2014). Impacto de la capacitación en el desarrollo de competencias en el profesorado universitario. Revista de Estudios y Experiencias en Educación, 13(25), 49-65. http://www.rexe.cl/ojournal/index.php/rexe/ article/view/47/49

González Serra, D. J. (2008). Psicología de la motivación. Ciencias Médicas.

Hair, J. F., Black, W. C., Babin, B. J., Anderson, R. E. y Tatham, R. L. (1999). Multivariate data analysis (5a ed.). Prentice Hall.

Juárez Adauta, S. (2012). Clima organizacional y satisfacción laboral. Revista Médica del Instituto Mexicano del Seguro Social, 50(3), 307-14. https://www.medigraphic.com/pdfs/imss/im-2012/im123n.pdf

Lorrén Cáceres, N. C. (2018). Clima organizacional y el desempeño laboral del personal de la empresa INPROCONSA, S.A.C en el primer semestre 2017 [Tesis de licenciatura, Universidad San Ignacio de 


\section{ABEH Y MUÑOZ PALOMEQUE}

Loyola]. Repositorio Institucional, Universidad San Ignacio de Loyola. http://repositorio.usil.edu.pe/ bitstream/USIL/3558/3/2018_Lorr\%C3\%A9n-Caceres.pdf

Lowenthal, W. (1981). Continuing education for professionals: voluntary or mandatory? The Journal of Higher Education, 52(5), 519-538. https://doi.org/10.1080/00221546.1981.11778122

Madrigal Torres, B. E. (2009). Habilidades directivas (2a ed.). McGraw-Hill.

Melo, N. A. P. (2020). El clima y la satisfacción laboral del capital humano. Innovar,30(76), 9-24. https:// doi.org/10.15446/innovar.v30n76.85191

Moncada Herrera, P. G. (2019). Motivación laboral y desempeño laboral en docentes universitarios de una institución educativa privada de Lima Metropolitana [Tesis de licenciatura, Universidad San Ignacio de Loyola]. Repositori Institucional, Universidad San Ignacio de Loyola. http://repositorio.usil.edu.pe/ handle/USIL/9754

Mujica de González, M. y Pérez de Maldonado, I. (2007). Gestión del clima organizacional: una acción deseable en la universidad. Laurus, 13(24), 290-304. https://www.redalyc.org/pdf/761/76111485014 .pdf

Paul, J. M. (2016). A study of relationships among teachers' perceptions of principal leadership and teachers' perceptions of school climate in the high school setting (Publicación $\mathrm{N}^{\circ}$ 3707889) [Tesis doctoral, University of Nevada, Reno]. ProQuest Dissertations and Theses Global.

Pedraza, E., Amaya, G. y Conde, M. (2010). Desempeño laboral y estabilidad del personal administrativo contratado de la Facultad de Medicina de la Universidad del Zulia. Revista de Ciencias sociales, 16(3), 493-505. https://doi.org/10.31876/rcs.v16i3.25519

Quintero, N., Africano, N. y Faría, E. (2008). Clima organizacional y desempeño laboral del personal Empresa Vigilantes Asociados Costa Oriental del Lago. Negotium, 3(9), 33-51. http://revistanegotium .org/pdf/9/Art2.pdf

Ramírez Amado, A. (2014). Capacitación y desempeño laboral de los empleados de la Universidad Linda Vista de la Unión Mexicana de Chiapas [Tesis de maestría, Universidad de Montemorelos, Montemorelos]. DSpace. http://dspace.biblioteca.um.edu.mx/xmlui/handle/20.500.11972/98

Rodríguez Cavanerio, L. V. y Coelho de Torres, B. G. (2018). Evaluación del desempeño de los docentes de la asignatura Histología y Embriología. Educación Médica Superior, 32(3), 181-194. http://www.ems .sld.cu/index.php/ems/article/view/1490/700

Rodríguez Horna, E. (2015). Relación entre clima organizacional, nivel de desempeño y motivación docente [Tesis de maestría, Universidad César Vallejo].

Rodríguez Montufar, G. (2020). Management skills that affect the organizational climate of higher education institutions. The Journal of Middle East and North Africa Sciences, 6(12), 1-9. http://oaji .net/articles/2020/2705-1606753097.pdf

Sallenave, J. P. (2010). Gerencia por resultados y planeación estratégica. Norma.

Sum Mazariegos, M. I. (2015). Motivación y desempeño laboral [Tesis de licenciatura, Universidad Rafael Landívar]. Libros en línea - Universidad Rafael Landívar. http://recursosbiblio.url.edu.gt/ tesisjcem/2015/05/43/Sum-Monica.pdf

Wu, Y., Lian, K., Hong, P., Liu, S., Lin, R.-M. y Lian, R. (2019). Teachers' emotional intelligence and selfefficacy: Mediating role of teaching performance. Social Behavior and Personality: An International Journal, 47(3), 1-10. https://doi.org/10.2224/sbp.7869 